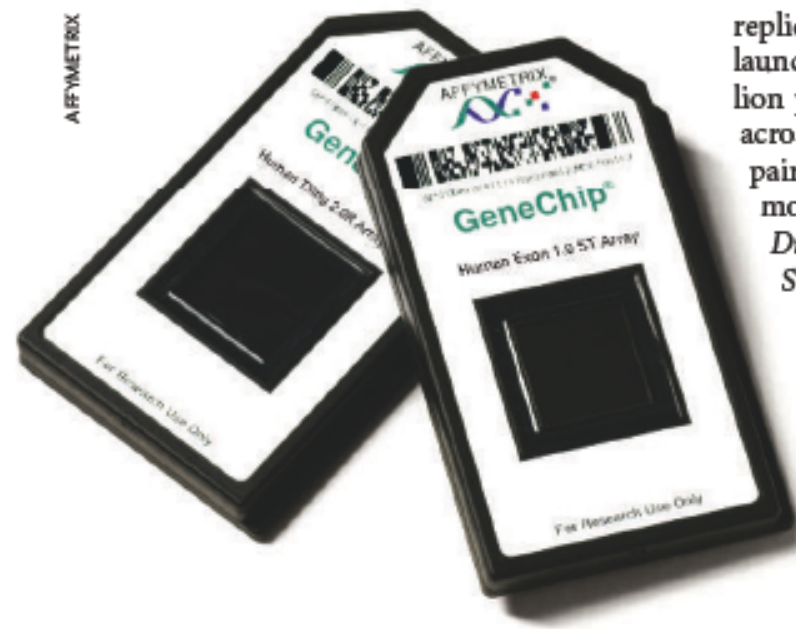

Looking close: Affymetrix's high-density tiling arrays interrogate the genome indetail.

we look across the entire genome without bias," says John Blume, vice-president of RNA products at Affymetrix.

The development of tiling arrays marked a departure from the way DNA microarrays had traditionally been designed. Rather than containing probes just for known or suspected functional regions, tiling arrays can be used to interrogate, or 'tile' across, the genome in an unbiased fashion, by means of probes spaced at regular intervals along the genome.

Tiling microarrays can be used for the genome-wide study of gene regulation, specifically to map sites of transcription-factor binding, chromatin modification, DNA methylation and chromosomal origins of replication. This month, Affymetrix will launch a set of seven arrays, each with $6.5 \mathrm{mil}$ lion probes and a $5-\mu \mathrm{m}$ feature size, to tile across the entire human genome at 35-basepair (bp) resolution. Tiling arrays for several model organisms, including Arabidopsis, Drosophila, Saccharomyces cerevisiae and Schizosaccharomyces pombe, are planned.

"Affymetrix will continue to innovate with respect to feature size. We don't think $5[\mu \mathrm{m}]$ is the end," says Blume.

The highest-density arrays from Agilent Technologies of Palo Alto, California, have 44,000 features. The company is about to launch its nextgeneration ink-jet printing platform for the non-contact in situ synthesis of 60-mer oligonudeotide probes on glass slides. With the new instrument, which will not be sold commercially, "we think that we can get about an order of magnitude higher density in the span of about two years," says Scott Cole, head of genomics marketing at Agilent.

\section{Scanning to size}

The ever-shrinking feature size on highdensity microarrays must also be coupled with improvements in scanning technology. This summer Affymetrix launched the new GeneChip Scanner 3000 7G with a scanning resolution in the sub-micrometre pixelation range, and which can scan features ranging in size from 2.5 to $0.51 \mu \mathrm{m}$. The scanner supports all high-resolution GeneChip products for tiling, all-exon and single-nucleotide polymorphism genotyping research.
For those on a budget, TeleChem International offers the $10-\mu \mathrm{m}$ resolution SpotLight microarray scanner. Rather than using lasers and photomultiplier tubes, as in most traditional scanners, the SpotLight uses 'cool' excitation technology that is optimized for two-colour detection (Cy3 and Cy5). Optional filter sets are available for fluorescein, rhodamine, allophycocyanin, ethidium bromide, the Alexa dyes and several green fluorescent protein variants. Scanning solutions are also available from established scanner manufacturers including arrayWoRxe from Applied Precision of Issaquah, Washington, GenePix from Molecular Devices of Sunnyvale, California and ProScanArray from Perkin Elmer.

CombiMatrix is developing an electrochemical detection (ECD) method to read its semiconductor-based microelectrode arrays, as an alternative to fluorescence. The company hopes to develop a smaller, less expensive detection system that will provide better performance than fluorescence-based systems the only method so far to be commercially successful. A prototype is in beta testing and a commercial product is expected next year. CombiMatrix hopes to corner the molecular diagnostics market with a handheld version.

Options are also available for researchers who want to print their own microarrays (see 'On the hardware front', page 1198).

\section{Comparative genomic hybridization}

For the best part of a decade, the microarray market has been dominated by gene expression. ${ }^{\alpha}$ But gene expression is not the most

\title{
MICROARRAYS MOVE DOWNSTREAM
}

\section{Array-based methods are now} being applied in a diagnostic setting and offer the possibility of analysing multiple analytes (and even multiple samples) in a highly parallel and high-throughput manner. In diagnosis, however, the tolerance for error is very low: it is important to get the right answer not just occasionally but all of the time, under stringent conditions and with varying sample quality. Nevertheless, important milestones have been achieved in the past year as array-based diagnostic tests begin to trickle onto the market.

Agendia of Amsterdam, The Netherlands, offers MammaPrint, a diagnostic test for as sessing the risk of breast cancer progression. The test predicts the future development of metastases on the basis of a 70-gene expression sign ature discovered by scientists at the Netherlands Cancer Institute and
Antoni van Leeuwenhoek Hospital in Amsterdam. Its use should improve the quality of treatment decisions for cancer patients by distinguishing those individuals who would, and who would not, benefit from further treatment.

In May, Tm Bioscience of Toronto, Canada, received FDA clearance for its Tag-lt cystic fibrosis (CF) kit to beused as an in vitrodevice for diagnostic use. The test, which identifies a group of mutations in the CF transmembrane conductance regulator (CFTR) gene, is based on the bead-based XMAP multiplexing technology developed by Luminex of Austin, Texas. XMAP allows multiplexing of up to 100 assays within a single sample. In addition to human genetic dis orders, Tm Bioscience is developing products aimed at pharmacogenetics and infectious diseases.

Last year, Affymetrix of Santa

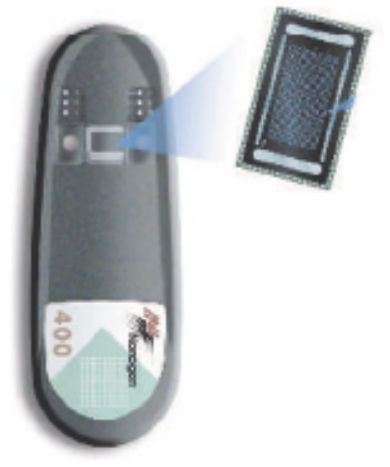

The NanoChipelectronic microarray goes diagnostic.

Clara, California, received clearance by regulatory authorities in the United States and Europefor the in vitro diagnostic use of its GeneChip System 3000Dx. Affymetrix's strategy for developing new arraybased diagnostic tests on its GeneChip platform is to work alongside partners from the diagnostics industry, such as Roche Diagnostics of Basel, Switzerland, bioMérieux of Marcy I'Etoile, France, and Veridex of Warren, New Jersey. "Weare a technology-driven company," says Robert Lipshutz, senior vicepresident of molecular diagnostics and emerging markets at Affymetrix." Wedid not havea large regulatory or commercial presence in the diagnostics industry."

The first microarray product to belaunched through the partnership with Roche is the AmpliChip CYP450 test, which identifies variations in two drugmetabolism genes that can affect the rate at which an individual metabolizes many common drugs.

Other companies are taking a different approach. CombiMatrix of Mukilteo, Washington, formed a diagnostic subsidiary, CombiMatrix Molecular Diagnostics, in May, now led by 
demanding thing you can do with a microarray," says Emile Nuwaysir, vice-president of business development at NimbleGen of Madison, Wisconsin. Other microarray applications are now coming to the fore "that even have the potential to eclipse gene expression", he says. One such area is array-based comparative genomic hybridization (CGH), which can provide a robust and accurate platform for routinely mapping chromosomal imbalances at exon-level resolution.

Several companies now offer microarraybased products and services for identifying and characterizing gains and losses of genomic regions that lead to copy-number changes in genes and regulatory regions. Such changes are thought to underlie diseases such as cancer and many congenital disorders. "To be able to see in the full complexity of the human genome that small change in signal is very demanding, and commercial arrays weren't capable of doing it five years ago," says Nuwaysir.

Unlike some array-based CGH products that use a gene-centric design, NimbleGen's human whole-genome array $\mathrm{CGH}$ platform is a single array with 385,000 probes tiled throughout the human genome every 6,000 bp. The company's customized fine-tiling $\mathrm{CGH}$ arrays contain the same number of probes as the whole-genome CGH array but tiled at higher resolution in chromosomal regions selected by the researcher. Here the probes can be spaced as densely as every $10 \mathrm{bp}$.

${ }^{\alpha}$ We're the only company that combines the benefits of high-density, long oligo arrays and the ability to change the design whenever you want," says Nuwaysir. NimbleGen also hopes that an isothermal probe-selection strategy will set it apart from the competition. Rather than fix the oligo length, as most companies do, NimbleGen fixes their melting temperatures at $76{ }^{\circ} \mathrm{C}$ across the entire set. As such, says Nuwaysir, the probes are optimized to perform equivalently in all genomic regions, including AT- and GC-rich regions.

Agilent introduced an updated version of its microarray-based CGH platform in August. The company already offers a microarray for the genome-wide scanning of chromosomal gains and losses on a single chip. Now, researchers will be able to tap into Agilent's database of approximately 4 million validated CGH probes and use its web-based DNA microarray design tool, eArray, to create custom CGH microarrays for high-resolution tiling of regions of interest. The company also recently launched a single-slide CGH microarray for the genome-wide profiling of DNA copy-number changes in mouse.

\section{Location, location, location}

A second expanding research area now being adapted to a microarray format is chromatin immunoprecipitation (chIP) analysis. Also referred to as 'location analysis', chIP followed by microarray analysis of the immunoprecipitated genomic DNA, the so-called chIP-chip methodology, can be used to identify the binding sites on chromosomes for DNA-binding proteins such as histones, polymerases and transcription factors, as well as studying DNA modification and chromatin-remodelling.

NimbleGen offers a microarray service for chIP-chip analysis. Researchers can either select from off-the-shelf array designs, which include a whole-genome survey set with 390,000 features per array and two array designs aimed at known promoter regions, or can create their own customized tiling chIP-chip arrays tailored to particular genomic regions. NimbleGen is also pushing the idea of array re-use, and currently guarantees three-time use of its chIP-chip products. "Our goal is to have a platform that is ten times re-usable in the next cycle," says Nuwaysir.

Agilent has also thrown its hat into this particular ring and launched its own chIP_ chip platform last month for analysing activity at regulatory genomic regions. In the future it expects to offer a variety of custom and catalogue ChIP-chip products, as the company acquired Computational Biology of Cambridge, Massachusetts, in January. This firm was founded by Richard Young of the Whitehead Institute at the Massachusetts Institute of Technology in Boston and has key intellectual property in this area. In a recent publication in Cell, Young's group used a chIP-chip approach to produce high-resolution genome-wide maps of acetylation and methylation in yeast.

\section{Splice-variant analysis}

Microarray analysis may have produced a mountain of gene-expression data but the contribution of alternative splicing has largely been overlooked until now. The market for former senior executives from the diagnostics industry. "We want to capture revenue not just from the chip but from the test it self," says Michael Tognotti, vice-president of sales and marketing at CombiMatrix.

"We didn't feel that highdensity arrays are the way to go for clinical utility," says Graham Lidgard, senior vice-president of research and development at Nanogen of San Diego, California. The company is targeting the clinical and diagnostic market with its NanoChip electronic microarray system. "If you want to do one or two targets, then reverse transcription-PCR is really the way to go, but if you want to look at four or five single-nucleotide polymorphisms to 100 targets across anumber of patients, our technology is probably best suited to that application," Lidgard says.

The company's NanoChip $\mathbf{4 0 0}$ platform for multiplexed mutation detection, out later this year, features increased automation and will offer a 400-site cartridge for increased throughput; the originalversion has 100 electrodes, or test sites.

An equity investment in Jurilab of Kuopio, Finland, announced in July, gives Nanogen certain rights to develop diagnostic products based on genes and gene markers discovered by Jurilab. The initial focus will be on cardiovascular and metabolic diseases, including adult-onset diabetes.

BioArray Solutions of Warren, New Jersey, has developed a novel twist on traditional bead-based systems where beads displaying proteins or DNA capture probes are in suspension and assays are performed 'in-tube'. BioArray's BeadChip format provides a bead array in the form of a singlepreassembled layer of colour-coded beads in random configuration on a smallsilicon chip. The company

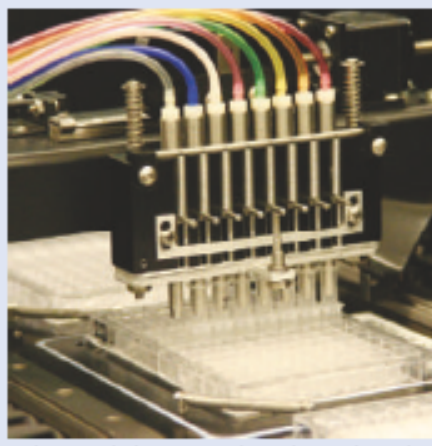

Micr ofluidics: Metrigenix's TipChip platform for RNA and DNA.

is initiallydeveloping BeadChip assays to screen carriers for CF and Tay-Sachs disease, as well as for transfusion and transplantation medicine.

"It's a very diverse set of applications that we've already developed and deliver on a complete platform," says Michael Seul, president of BioArray Solutions. "As a small company, we've chosen to focus on certain areas where we can have a large impact because they have been largely unexplored."

MetriGenix of Toronto, Canada, is developing genomic biomarker panels and genomics instrumentation for the diagnostic market. In the company's Flowthru chip technology, molecular interactions occur within 3D microchannels rather than on a planar surface.

The company's TipChip technology is provided as part of the MGX8 automated system, which can process eight $1 \times 1-\mathrm{cm}$ silicon TipChips in parallel with up to 1,600 biomolecular targets or 'spots' per chip. The system is suitable for RNA, DNA or protein targets.

Sample preparation is still a time-consuming process in all platforms. Further automation on the front end would improverobustness and reduce costs. 
splice arrays is now heating up, and companies such as ExonHit of Paris, France, and Jivan Biologics of Berkeley, California, are focused entirely in this area.

Most human genes are thought to undergo alternative splicing, the process by which individual genes can produce multiple messenger RNA and protein products. These socalled splice isoforms can have different, and even opposing, functions in the cell. With the recent commercial availability of splice-variant arrays, traditional gene-by-gene studies of alternative splicing are now taking a back seat to genome-wide methods. Further study in this area should provide a better - and more complete - understanding of the functional relevance of splice variants and of disease mechanisms.

ExonHit offers its SpliceArrays on a service basis, but from this September they can also be purchased directly from Agilent. Validation of the ExonHit approach, which involves the use of both exon- and junction-derived probes to follow every splice event associated with a given gene, was featured in a recent publication in Nucleic Acids Research.

The company offers both custom and catalogue SpliceArrays focused on specific gene families that are of particular therapeutic interest - cytokine and apoptosis pathways, nuclear receptors and co-regulators, Gprotein-coupled receptors (GPCRs) and ion channels. "We are also in the process of expanding our line into mouse," says Laurent Bracco, ExonHit's vice-president of research and technology development, with products

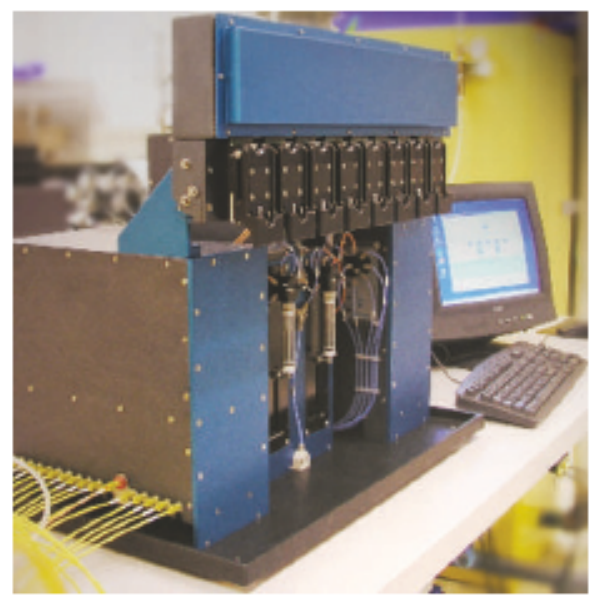

Desktop DNA synthesis from CombiMatrix.

expected around the turn of the year.

Jivan has been working in this area for the past four years and offers its suite of TransExpress splice-variant microarrays, which are manufactured by Agilent. The company's products include a genome-wide splice-variant array and a suite of arrays focused on gene families such as cytochrome P450, GPCRs, ion channels, kinases, phosphatases, phosphodiesterases, proteases and proteinases. Unlike ExonHit, Jivan's splice arrays do not include exon probes. "Exon probes are neither necessary nor sufficient to measure RNA splicing and so we do not include them in our Trans Express product line. Using junction probes, however, all splicing events can be detected," says Jonathan Bingham, Jivan's chief technol- ogy officer. The company also makes arrays to $x$ user specifications, and now offers a splicevariant microarray service through MOgene of St Louis, Missouri. "The next product will 8 be an oncology array consisting of about 2,000 genes implicated in cancer along with their splice variants," says Bingham.

At the high-density end of the market, earlier this month Affymetrix rolled out its exononly array with a million exons on a single chip. This Human Exon 1.0 ST (sense target) array offers whole-genome, exon-level expression profiling on a single array and includes all annotated exons, as well as computationally predicted and empirically identified exon content. As such, the company says, researchers will be able to study known splicing events, as well as discover novel splice variants. GeneChip mouse and rat exon arrays are planned for later this year.

Microarrays are now a widely used technology for studying gene expression and regulation on a global scale and at high-throughput. Other applications for this technology include single-nucleotide polymorphism discovery and validation (see Nature, 422, 917-922; 2003) and comparative genome sequencing. The use of microarrays is also moving downstream as the first microarray-based diagnostic tests begin to trickle into the marketplace (see 'Microarrays move downstream,' page 1196). The future indeed looks bright. Has it really been only ten years?

Diane Gershon is assistant editor, Nature Medicine, Technical Reports
Researchers interested in printing their own microarrays in-house now have an array of options from contact (pin or split pin) and non-contact printing methods (bubblejet or piezoelectric inkjet) to in situ array synthesis.

Bio-Rad Laboratories of Hercules, California, launched the BioOdyssey Calligrapher miniarrayer last month, which prints oligos, proteins or cell lysates onto slides, membranes or 96-well plates. This bench-top instrument has an eight-pin print head and prints up to 16 slides at a time. Optional extras include humidity and temperature controls. Telechem International of Sunnyvale, California, has also added humidity controls to its NanoPrint microarrayer.

A plate-arraying option is now available on the BioRobotics MicroGrid ii and GeneMachines
OmniGrid Accent platforms from Genomics Solutions of Holliston, Massachusetts. This enables DNA and protein microarrays of up to 1,000 features per well to be printed in 96- and 384-well microplates, providing highthroughput, multiplexing capabilities for diagnostic, ELISA and protein-protein interaction applications.

Non-contact printers may have got a bad rap in the early days but Arrayjet of Mayfield, UK, hopes to win over researchers with its new Aj120 piezoelectric inkjet printer, which it launched in July. "Contact printing can affect the quality of the data you get because it introduces surfacebased artefacts into the microarray," says Duncan Hall, sales and marketing director for Arrayjet. Pin printers can also be very susceptible to atmospheric conditions, he says, particularly

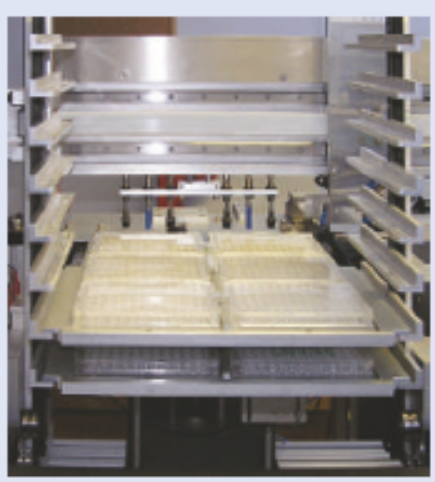

Arrayjet makes non-contact printing high-throughput.

when hygros copic printing buffers are used. "We carry our sample inside the print head so there isn't anyevaporation from the print head."

The Aj120 includes a microplate stacker and lid lifter, which enables walk-away printing of microarrays from up to 48 ( $96-$ or 384-welD microtitre plates. With the standard 12-sample connector block, 100 slides (samples and replicates side-by-side) can beprinted in 90 seconds, says Hall. Additional replicates can be printed on the fly in no extra time. The Aj120 prints DNA, proteins and intact cells with a 100- $\mu \mathrm{m}$ spot diameter. PerkinElmer Life and Massachusetts, also offers contact and piezoelectric non-contact systems.

For those with deeper pockets, CombiMatrix sells aCustomArray desktop DNA synthesizer for the in situ synthesis of oligonucleotides on microarrays with up to 12,000 features.Synthesis occurs on a blank CombiMatrix semiconductor chip using standard phosphoramidite chemistry methods. AnalyticalSciences of Boston, 